\title{
Investigation of the fatigue behavior of notched reinforced thermoplastics
}

\author{
Ilan Raphael ${ }^{1,2,3^{*}}$, Nicolas Saintier ${ }^{1}$, Lucien Laiarinandrasana ${ }^{2}$ and Gilles Robert $^{3}$ \\ ${ }^{1}$ I2M, Arts et Métiers Paris Tech, Esplanade des Arts et Métiers, 33405 Talence, France \\ ${ }^{2}$ MINES Paristech, Centre des Matériaux, BP 87, 91003 Evry Cedex, France \\ ${ }^{3}$ Solvay Engineering Plastics, Avenue Ramboz, BP 64, 69192 Saint-Fons, France
}

\begin{abstract}
Understanding and modeling of fatigue damage and behavior in short glass fiber reinforced thermoplastics is an important issue in order to improve material processing and the design of composite parts for the industry. The present work aims at a more precise description of the fatigue damage mechanisms and the establishment of a simple, yet efficient, durability criterion. Observations bring new insights on the fatigue damage mechanisms: fiber failure triggered by fretting fatigue and crack propagation by intra-spherulitic failure in their equatorial plane. Finally, a cyclic strain rate criterion is introduced for notched specimens and the identification of a behavior model is performed for later implementation of the durability criterion.
\end{abstract}

\section{Introduction}

\subsection{Context of the study}

In order to comply with new legislation regarding the environment, manufacturers in the car industry need to reduce the weight of parts and structures. One way to do so is to use composite materials rather than metals. Short glass fibers reinforced (SGFR) thermoplastics constitute a promising category of material thanks to their good specific mechanical properties and ease of manufacturing as injection molding allows for high production rates as needed in the car industry.

Short glass fiber reinforced polyamide 6,6 (PA66) is increasingly used, including for crucial components of the vehicle. However in order to engineer more efficient parts, a better knowledge of the mechanical properties of the material is required, especially regarding its durability under fatigue loading. Advances are needed both in our understanding of the damage mechanisms and the modeling of the behavior at the macro and micro scales.

Previous studies have established damage scenarios under quasi-static and fatigue loading using fractography analysis and in-situ tomographic observations $[1,2]$. The aim is now to refine our knowledge of these mechanisms, especially at the onset of fatigue damage.

\subsection{Material}

\subsubsection{Fiber orientation}

In injection molded parts, the short glass fibers are oriented following a well described core-shell microstructure [3]. In the shell fibers are aligned with the mold flow direction (MFD). Conversely, in the core at the center of the plate, the fibers are aligned perpendicularly to the MFD.

Specimens are usually described as $0^{\circ} / 45^{\circ} / 90^{\circ}$ following the direction of their axis with respect to the MFD.

\subsubsection{Spherulitic structure}

Semi-crystalline thermoplastics often crystallize as spherulites [4]. These are spherical structures nucleated from a single lamella and composed of a succession of crystalline lamellae and amorphous polymer chains. The width of a lamella and the distance between two lamellae is of about $10 \mathrm{~nm}$.

Spherulite diameter depends on the cooling conditions and number of potential nucleating sites. In our material spherulite diameter is generally comprised between 15 and $30 \mu \mathrm{m}$.

\subsection{Notched specimens}

Specimens are waterjet-cutted from injection-molded rectangular plates $3.24 \mathrm{~mm}$ thick. Two notch geometries were considered: symmetric "U" notches $0.5 \mathrm{~mm}$ in radius and symmetric " $U$ " notches $2 \mathrm{~mm}$ in radius. For pure PA66, specimens were all cut at $90^{\circ}$. For reinforced PA66, specimens are designated by the direction of the fibers in the shell with respect to the specimen main axis.

The use of notched specimens allows for the localization of the damage. This is useful in order to facilitate the

\footnotetext{
*Corresponding author: ilan.raphael@ensam.eu
} 
observations of fatigue damage. Furthermore, the different notch geometries result in different local mechanical fields and different gradients of those mechanical fields.

Specimens were conditioned at RH50 prior to testing and experiments were conducted at $23^{\circ} \mathrm{C}$. Fatigue loading was performed at $0.5 \mathrm{~Hz}$ and at a loading ratio $\mathrm{R}=0.1$.

\section{Fatigue damage mechanisms}

\subsection{Usual description}

Fatigue damage scenarios traditionally include cavitation and crazing in the polymer matrix, damage at fiber tip, debonding at the fiber-matrix interface and fiber failure.

These mechanisms can be observed on the fracture surface using a scanning electron microscope (SEM) or in the bulk of the material by performing X-ray tomography (fig. 1).

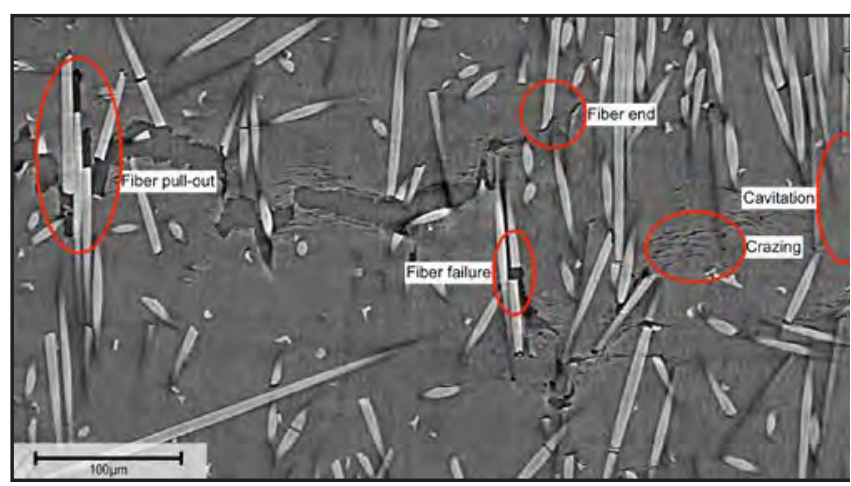

Fig. 1. Fatigue damage mechanisms observed by tomography.

\subsection{On the importance of cavitation}

Cavitation occurs both in poorly reinforced areas where local strains can be high and close to fibers where confinent is important. Based on in-situ observations and fractography analysis, cavitation in the thermoplastic matrix appears as a precursor of other fatigue damage mechanisms.

Cavitation in the matrix is an early sign of damage that subsequently can lead to the formation of craze and short cracks is the material.

Debonding at fiber-matrix interface is generally described as starting from the fiber tips, where adhesion is poor. However cavitation along fibers can also in turn induce a weakening of the interface (fig.2). This might lead to a local stress redistribution triggering a failure of the fiber.

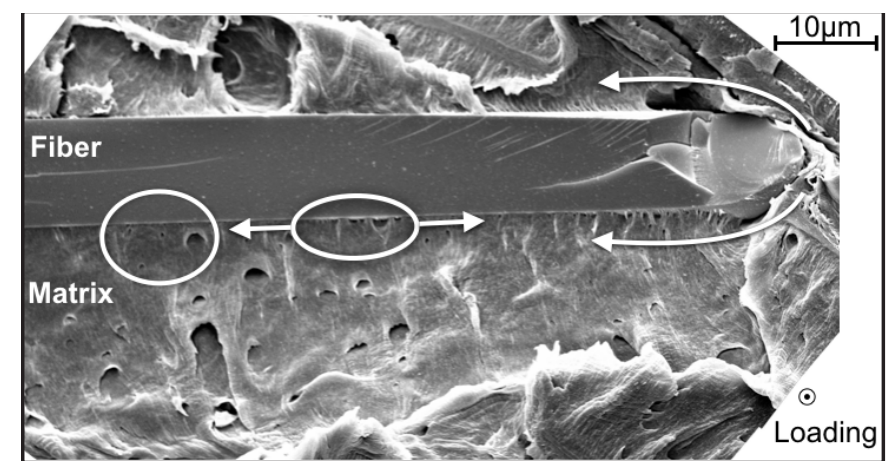

Fig. 2. Damage at the interface induced both by the poor bonding at the fiber tip and cavitation close to the interface.

\subsection{Fiber failure by fretting fatigue}

Failure of the short glass fibers is one of the best known damage mechanisms in SGFR thermoplastics. In quasistatic tensile tests, fibers break because they are overloaded. Yet in fatigue multiple scenarios can lead to the breakage of glass fibers.

Earlier works have shown that fibers tend to break at the crossing with an other fiber. This crossing of fibers induces a confinement of the matrix in between and a stress concentration in the adjacent fibers, thus triggering the failure (fig.3).

However, in some cases, fibers are in contact. This can lead to indentation and/or fretting fatigue between the fibers. The reinforcement being weakened, its failure become much more likely. This fiber failure triggered by contact between reinforcements in the composite material has, to our knowledge, not been described in previous damage scenarios, yet is can be unambiguously observed by fractography analysis (fig.4).

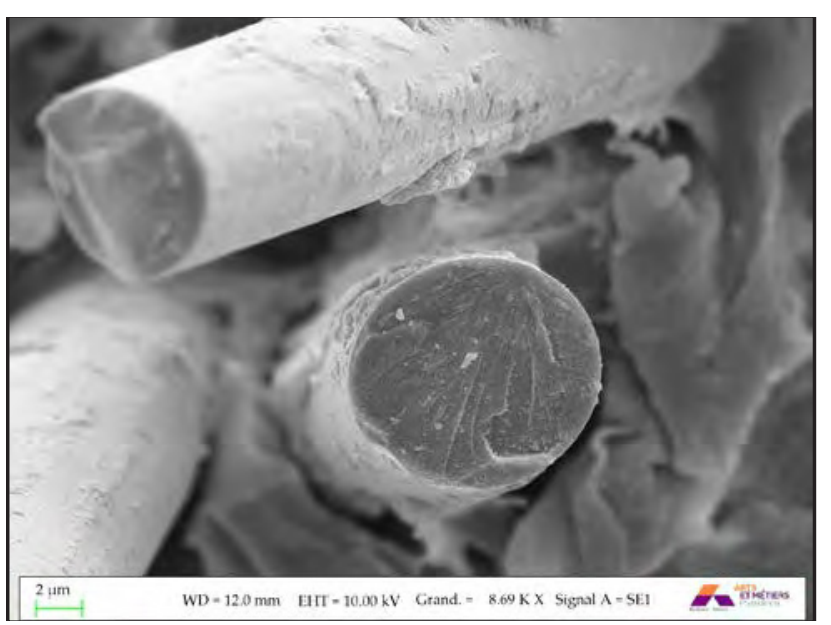

Fig. 3. Failure induced by a crossing with a neighboring fiber. 


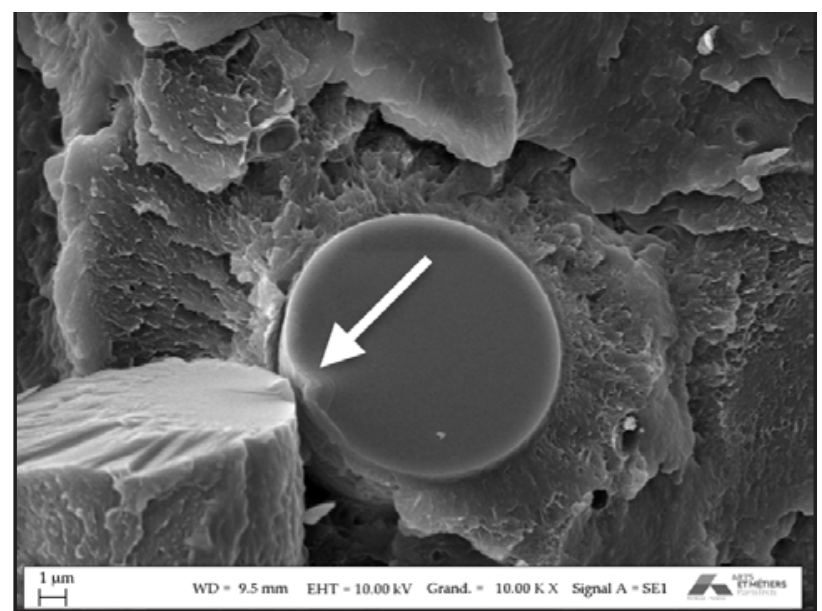

Fig. 4. Failure induced by the contact with a neighboring fiber.

\subsection{Spherulitic fatigue damage}

\subsubsection{Tomographic observations}

In-situ tomographic observations have revealed that damage progresses in the matrix with a characteristic length of $20-25 \mu \mathrm{m}$, close to the spherulite diameter.

Further analysis reveal that these structures are flat penny-shaped disks, that could correspond to the equatorial planes of spherulites.

Subsequently a damage scenario was advanced for damage in the semi-crystalline polymer matrix: fatigue damage progresses by opening the spherulites in their equatorial plane.

In order to confirm such a hypothesis, fractography observations have been performed.

\subsubsection{Fractography observations}

SEM observations on the fracture surface of notched unreinforced PA66 specimens reveal a ductile layer in the fatigue crack path.

In this area, it is possible to clearly observe spherulites. The radial organisation of the lamellae is visible, as well as the nucleus of the spherulite (fig.5). This confirms the hypothesis of intra-spherulitic failure in the equatorial plane.

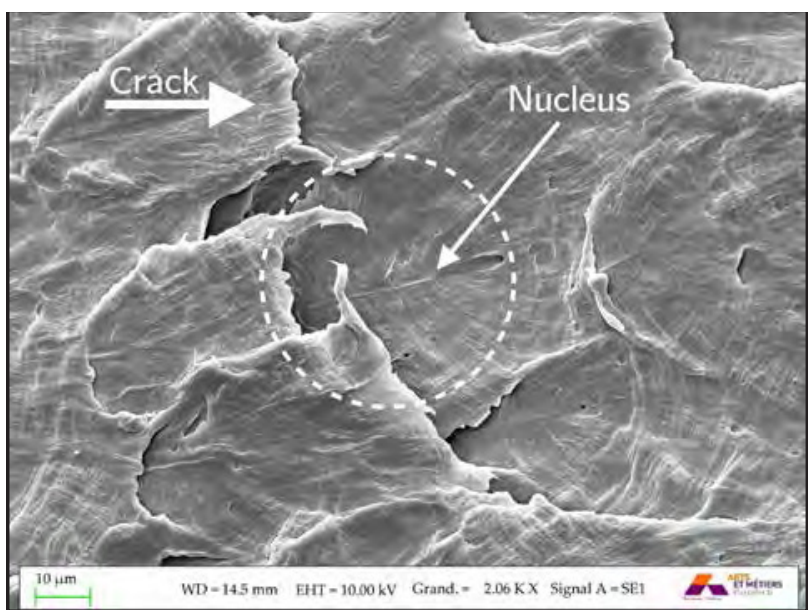

Fig. 5. Spherulites as observed on the fracture surface of PA66 after fatigue loading.

\section{Modeling of fatigue behavior}

\subsection{Towards a pertinent fatigue indicator}

\subsubsection{Cyclic strain rate in secondary state}

In order to unify fatigue results for SGFR PA66, one should determine a pertinent indicator that yields consistent results for the multiple conditions that can be encountered. These conditions can vary greatly with the imposed loading, the structural heterogeneities of the part or the environment. Fatigue results for various fiber orientations, mean stresses, loading ratios, frequencies, notches and relative humidity should all be unified by a pertinent indicator.

Previous results on smooth specimens have shown that the cyclic strain rate during the secondary state of loading unifies fatigue results. This cyclic strain rate can be determined by measuring the slope of the Mean strain per cycle vs. Number of cycles curve at half life of the specimen (fig. 6). 


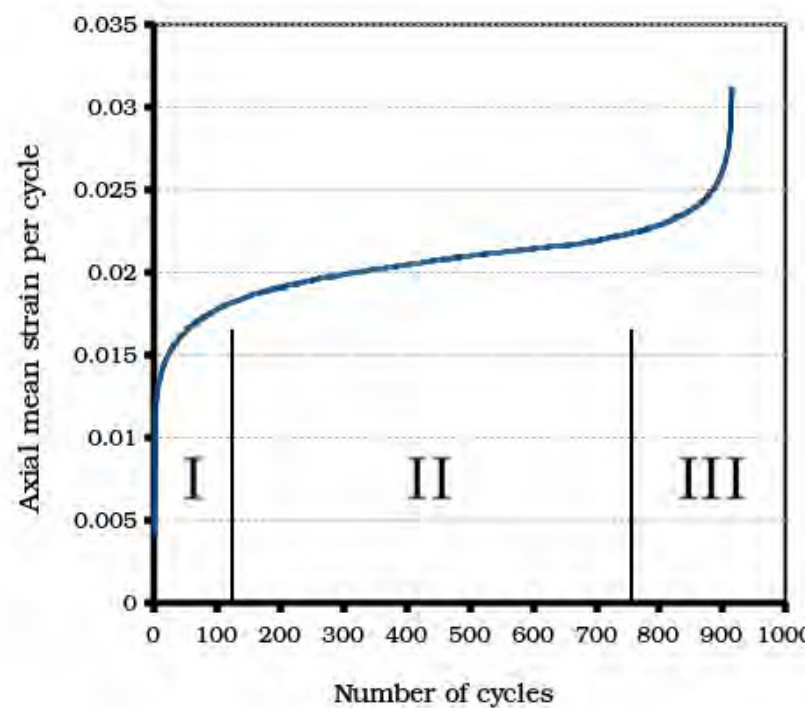

Fig. 6. Mean strain per cycle during the fatigue loading of a pure PA66 specimen.

\subsubsection{Experimental results on notched specimens}

Fatigue testing on notched SGFR PA66 specimens have shown that the cyclic strain rate at mid-life is also a good indicator. Is can be used to identify a durability criterion:

$$
N_{r}=A\left(\frac{\dot{\epsilon}_{11}}{\dot{\epsilon}_{0}}\right)^{n}
$$

with the following parameters: $A=0.220$ and $n=-0.81$.

It yields a good estimation of $\mathrm{Nr}$, the number of cycles until failure, regardless of fiber orientation and notch geometry (fig.7).

\subsubsection{Measuring a local strain rate}

In order to obtain a criterion that can be implemented in a Finite-Element (FE) model, more data is needed regarding the local cyclic strain rates close to the notch. An image acquisition setup, timed with the loading cycles, has been put in place. Images of the specimen are taken every 20 cycles at peak loading.

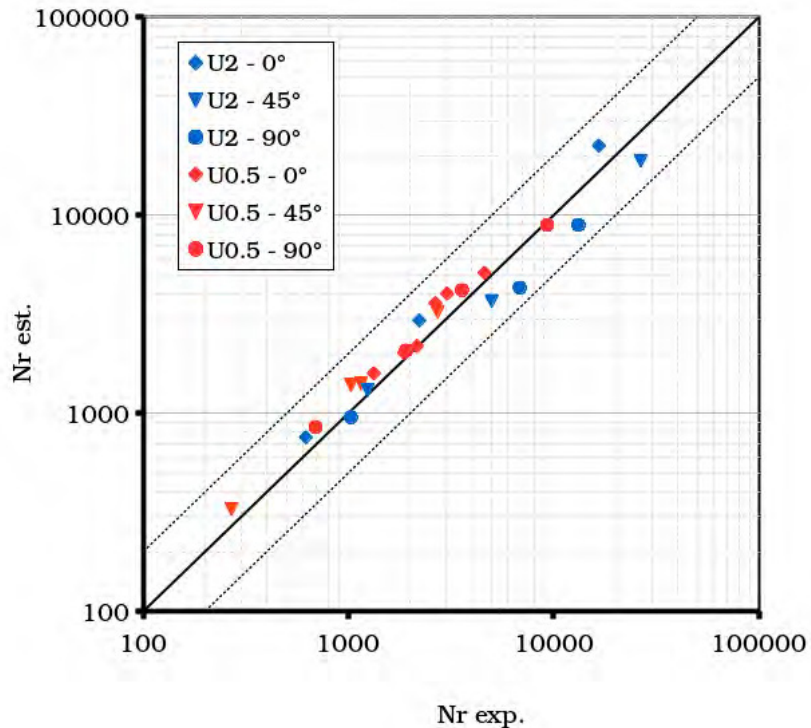

Fig. 7. Durability criterion based on the macroscopic cyclic strain rate.

Digital image correlation (DIC) can then be performed using VIC-2D in order to determine local cyclic strains and strain rates. These values can be compared to those obtained by modeling.

The local data can also be used in order re-identify the parameters of the equation (1) based on the local axial strain rate at notch tip giving $A=19.55$ and $n=-0.421$. These can thus be used as a new local durability criterion (fig.8).

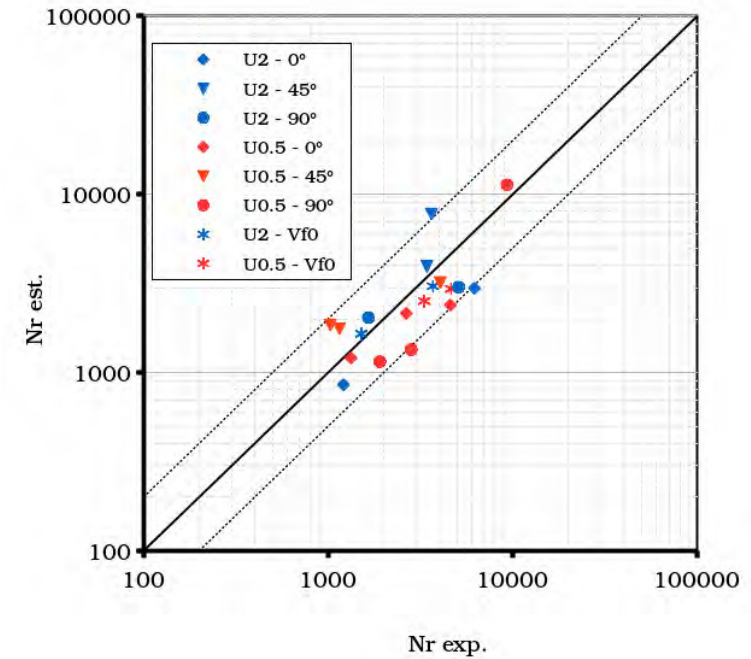

Fig. 8. Durability criterion based on the macroscopic cyclic strain rate.

The criterion unifies fatigue results for different notch geometries, fiber orientation as well as for unreinforced PA66 notched specimens. 


\subsection{Modeling}

\subsubsection{Constitutive equations}

Total strain is parted between a linear elastic contribution and a visco-plastic contribution:

$$
\epsilon=\epsilon^{e}+\epsilon^{v p}
$$

Plastic strain rate is defined using Norton law:

$$
\dot{p}=\left(\frac{\phi}{K}\right)^{n}
$$

with:

$$
\phi=\sigma_{*}-R(p)
$$

and the isotropic hardening given by:

$$
R(p)=R_{0}+Q(1-\exp (-b p))
$$

The GTN model can be used to calculate the effective stress $\sigma_{*}$ by taking into account the growth of cavities in the material. This volume fraction of cavities $f_{*}$ is used as the damage variable.

Thus $\sigma_{*}$ is calculated using:

$$
\Phi\left(\Sigma, f_{*}, \sigma_{*}\right)=\frac{\Sigma_{e q}^{2}}{\sigma_{*}^{2}}+2 q_{1} f_{*} \cosh \left(\frac{q_{2} \Sigma_{k k}}{2 \sigma_{*}}\right)-1-q_{1}^{2} f_{*}^{2}=0
$$

\subsubsection{Parameter identification}

Parameter identification on notched specimens have first been performed using a genetic algorithm on quasi-static tests at $\dot{\epsilon}=10^{-2} S^{-1}$ and $\dot{\epsilon}=10^{-3} S^{-1}$ in order to match the stress-strain curves and the notch opening displacement (NOD).

Parameters have then been adjusted for the cyclic loading. Focus was primarily set on obtaining a consistent value for the cyclic axial strain rate in order to later be able to apply the durability criterion (see Eq.1).

\subsubsection{Results for quasi-static tensile loading on PA66}

Quasi-static tensile tests were performed at two strain rates $\dot{\epsilon}=10^{-2} S^{-1}$ and $\dot{\epsilon}=10^{-3} S^{-1}$. Load and NOD are plotted and compared to the experimental data (fig.9 and fig.10).

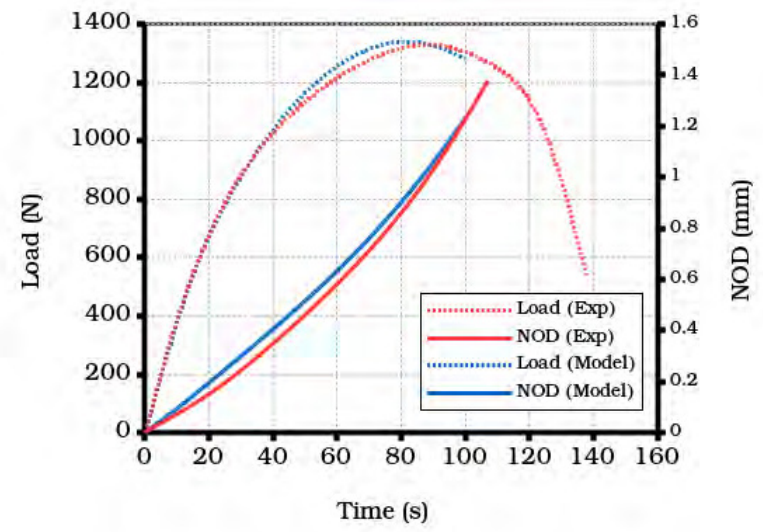

Fig. 9. Load and NOD for a U2 notched PA66 specimen during tensile testing performed at $\dot{\epsilon}=10^{-3} S^{-1}$.

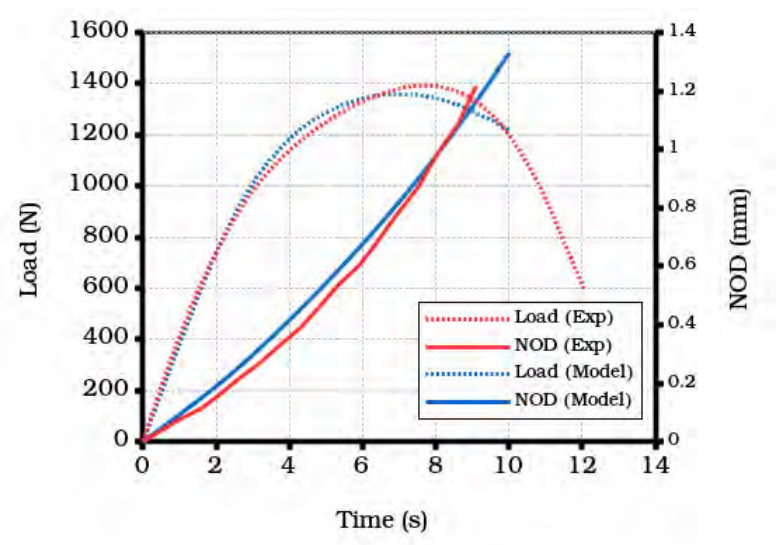

Fig. 10. Load and NOD for a U2 notched PA66 specimen during tensile testing performed at $\dot{\epsilon}=10^{-2} S^{-1}$.

\subsubsection{Results for cyclic loading on PA66}

First cyclic tests were performed at $0.5 \mathrm{~Hz}$ and $\mathrm{R}=0.1$. Axial strain is averaged on each cycle to be plotted and compared to the experimental data (fig.11 and fig.12).

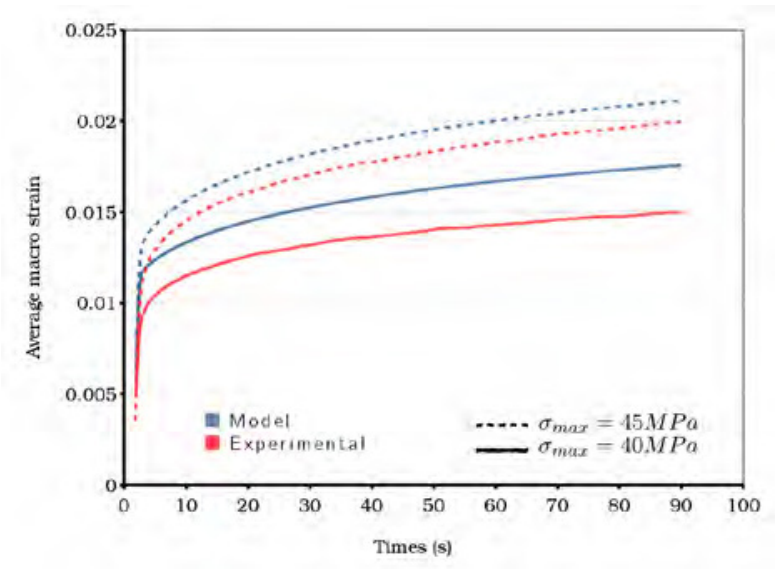

Fig. 11. Average axial strain for a U2 notched PA66 specimen. 


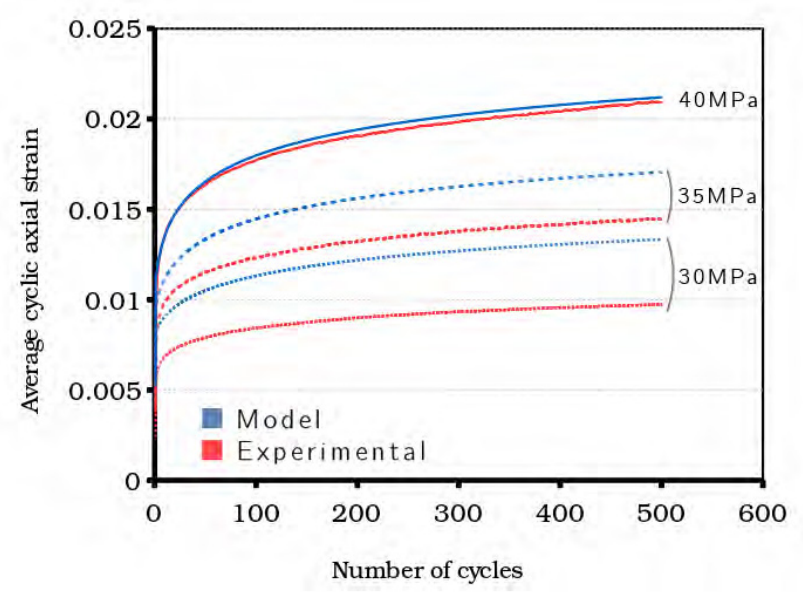

Fig. 12. Average axial strain for a U0.5 notched PA66 specimen.

It can be noted that the average axial strain differs from the experimental data, especially at lower stress levels. However, in all cases, the strain rate in accurately determine as the slopes of the experimental and model curves are alike.

The computed NOD can also be compared to the experimental data (fig.13), as well as the cyclic strain rate between notches at half the fatigue life of the specimen (fig.14), giving a good agreement between the model and the experimental results.

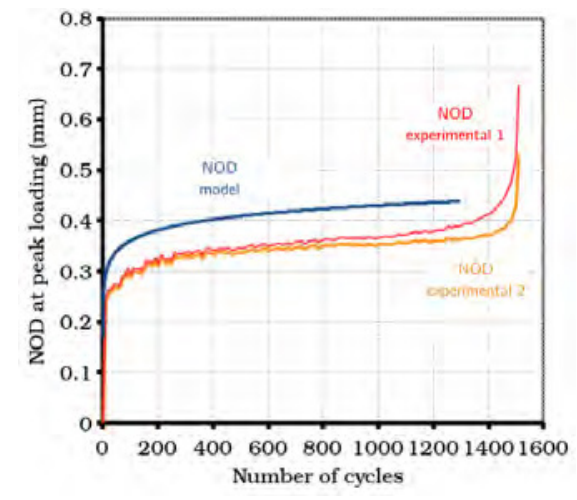

Fig. 13. NOD for a U2 notched PA66 specimen during fatigue loading.

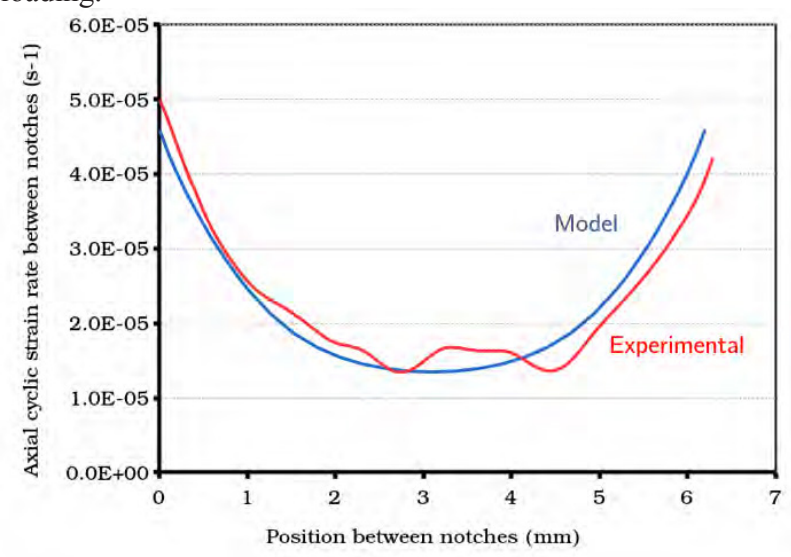

Fig. 14. Axial cyclic strain rate between the notches for a U2 notched PA66 specimen.

\section{Conclusion}

Our fractography analysis and tomographic observations have shed light on the importance of better understanding fatigue damage mechanisms. Observations have thus shown that cavitation is a precursor of other fatigue damage mechanisms, and in particular that it can lead to a damage of the fiber-matrix interface resulting in the breakage of the fiber. Fractography analysis also showed that fiber failure can be triggered by the contact between crossing fibers, resulting in a weakening of the reinforcement by fretting fatigue. Moreover, analysis of the fracture surface of pure PA66 specimens showed that fatigue damage progress is closely linked to the semi-crystalline microstructure of the polymer. Further work should evaluate the impact of the spherulitic structure, size and anisotropy at a finer scale on the fatigue damage progress and localization.

Experimental results on notched specimens have shown that a simple two-parameters cyclic strain rate criterion can efficiently unify fatigue results for different fiber orientations, notch geometries and loading intensities. The criterion can be tested and later improved, for example by considering an equivalent strain and/or a non-local measurement around the notch tip.

A model has been identified that can match the experimental cyclic strain rate. The model should now be tested on various conditions of loading and notch geometries.

\section{References}

1. Horst JJ. PhD. 1997.

2. Rolland H, Saintier N, Raphael I, Lenoir N, King A, Robert G. Compos Part B Eng. (2018);143:217-229. doi:10.1016/j.compositesb.2017.12.051.

3. Arif MF. PhD. 2014.

4. Crist B, Schultz JM. Prog Polym Sci. (2016);56:1-63. doi:10.1016/j.progpolymsci.2015.11.006. 